\title{
Frontières
}

\section{La construction sociale des morts attribuées à la drogue}

\section{Augustinus A.N. Cruts}

Volume 14, numéro 1, automne 2001

Où est la marge?

URI : https://id.erudit.org/iderudit/1074145ar

DOI : https://doi.org/10.7202/1074145ar

Aller au sommaire du numéro

Éditeur(s)

Université du Québec à Montréal

ISSN

1180-3479 (imprimé)

1916-0976 (numérique)

Découvrir la revue

Citer cet article

Cruts, A. A. (2001). La construction sociale des morts attribuées à la drogue. Frontières, 14(1), 14-17. https://doi.org/10.7202/1074145ar

\section{Résumé de l'article}

Dans cet article, je vous invite à regarder les morts attribuées à la drogue du point de vue du constructionnisme social. On accuse souvent cette théorie de nier les faits, surtout qu'il demeure indéniable qu'il existe des doses mortelles de substances légales et illégales. Sous cet angle, c'est un truisme de dire que les drogues tuent. Néanmoins, l'attribution de la principale cause d'un décès à une certaine drogue est une construction sociale. Le cas qui sera analysé ici démontre qu'une mort attribuée à la drogue aurait tout aussi bien pu être attribuée au libre marché. Il est certain que le libre marché tue autant que les drogues. L'argument qui sera mis en valeur est que la mort attribuée à la drogue est une construction sociale parce qu'une telle conclusion se fonde sur un raisonnement invérifiable dans les faits. Ce recours à des raisonnements invérifiables dans les faits pour imaginer un monde sans drogues peut être considéré comme l'expression d'un point de vue personnel sur la vie.
Ce document est protégé par la loi sur le droit d'auteur. L'utilisation des services d’Érudit (y compris la reproduction) est assujettie à sa politique d'utilisation que vous pouvez consulter en ligne.

https://apropos.erudit.org/fr/usagers/politique-dutilisation/ 


\section{Résumé}

Dans cet article, je vous invite à regarder les morts attribuées à la drogue du point de vue du constructionnisme social. On accuse souvent cette théorie de nier les faits, surtout qu'il demeure indéniable qu'il existe des doses mortelles de substances légales et illégales. Sous cet angle, c'est un truisme de dire que les drogues tuent. Néanmoins, l'attribution de la principale cause d'un décès à une certaine drogue est une construction sociale. Le cas qui sera analysé ici démontre qu'une mort attribuée à la drogue aurait tout aussi bien pu être attribuée au libre marché. Il est certain que le libre marché tue autant que les drogues. L'argument qui sera mis en valeur est que la mort attribuée à la drogue est une construction sociale parce qu'une telle conclusion se fonde sur un raisonnement invérifiable dans les faits. Ce recours à des raisonnements invérifiables dans les faits pour imaginer un monde sans drogues peut être considéré comme l'expression d'un point de vue personnel sur la vie.

Mots clés: constructionnisme social mort attribuée à la drogue

\begin{abstract}
This article invites you to a social constructionist view on the issue of drug-related death. Social constructionism is often misunderstood for denying plain facts. It sure is a fact that there are deadly doses of legal and illegal substances. In this sense it is a truism that drugs kill people. Nonetheless, it is argued that reducing the causes of death to a certain drug as the essential underlying cause of death is a social construction. A case is discussed to demonstrate that a drug-related death can just as well be seen as a free-market death. Free markets kill people at least as much as drugs do. It is argued that drugrelated death is a social construction, because attributing a death to a drug is based on unfalsifiable counterfactual thinking. Counterfactual thoughts about what the world would look like if there were no drugs, are seen as expressing one's view of life.
\end{abstract}

Key words : social constructionism drug-related death

$\begin{array}{lllllll}A & R & T & \text { I } & \text { C } & \text { L } & E\end{array}$

LA CONSTRUCTION

SOCIALE DES MORTS ATTRIBUÉES À LA DROGUE

\section{Augustinus A.N. Cruts ${ }^{1}$, Trimbos Institute, Utrecht, Pays-Bas.}

Traduit par Suzanne Mineau².

Le constructionnisme social est un mouvement postmoderne de la philosophie des sciences. Gergen (1999, p. 228), qui est considéré comme l'une des grandes figures de ce mouvement, le définit comme «une invitation à une danse, un jeu, une conversation ou une forme de vie». À sa suite, je vous invite donc à considérer d'un point de vue constructionniste une question aussi grave que la mort attribuée à la drogue.

Bien des auteurs soutiennent que les drogues illégales sont une construction sociale, par exemple Cohen (1990), Van de Wijngaart (1991), DeGrandpré (1996), Schneider (1996), Davies (1997) et Lyons et Rittner (1998). Selon eux, aucune raison scientifique objective ne peut expliquer pourquoi une substance comme l'alcool est légale alors qu'une autre comme le cannabis est illégale. Aucune loi physique n'impose la mise sous tutelle d'une drogue. Son caractère légal ou illégal est un accident de l'histoire.

Si les drogues illégales sont une construction sociale, la mort attribuée à la drogue est évidemment une construction sociale elle aussi. Legnaro (1997) était déjà arrivé à la même conclusion et jusqu'ici, je me suis contenté de présenter de vieilles idées sous une forme nouvelle. Ce qu'il y a de novateur dans mon analyse est l'argument suivant: une mort attribuée à la drogue est une construction sociale parce qu'une telle conclusion s'appuie sur un raisonnement invérifiable dans les faits. Un raisonnement peut être validé dans les faits lorsque l'absence de ces faits change automatiquement la conclusion. En d'autres mots, en attribuant à une drogue illégale la mort de quelqu'un, on présuppose que cette personne serait encore vivante s'il n'y avait pas eu cette drogue. On ne pourra jamais prouver de façon empirique la véracité de ce raisonnement parce qu'on ne peut pas valider empiriquement la proposition contraire. C'est pourquoi les idées d'une collectivité au sujet de la cause sousjacente à une mort sont des constructions sociales.

Dans le présent article, je me propose tout d'abord, afin d'éviter tout malentendu, de réfuter les critiques selon lesquelles une construction sociale est une négation irréaliste des faits, telle l'existence de doses mortelles de drogues. Pour concrétiser davantage mon raisonnement, je présenterai l'histoire d'une mort attribuée à la drogue. À partir de ce cas, je montrerai ensuite qu'une mort attribuée à la drogue est une construction sociale parce qu'elle pourrait tout aussi bien être attribuée au libre marché. En d'autres mots, je montrerai que le libre marché tue au moins autant que les drogues.

\section{FAITS INDÉNIABLES}

Lorsque Gergen (1999, p. 222) demandait à ses interlocuteurs au cours d'une discussion d'adopter le point de vue du constructionnisme social, il devait constamment faire face à un sentiment frustrant d'incrédulité. "Comment pouvez-vous remettre en question l'existence d'une réalité matérielle? lui demandaient ses interlocuteurs incrédules. Essayez-vous de nous dire que la pollution, la pauvreté ou la mort ne sont pas réelles? Voulez-vous dire que le monde n'existe pas, que nous ne faisons que l'inventer? C'est absurde! » 
LA MORT PEUT ÊTRE ATTRIBUÉE À BIEN DES CAUSES.

RÉDUIRE TOUTES CES CAUSES À UNE SEULE

EN FAISANT D'UNE DROGUE ILLÉGALE

LA CAUSE SOUS-JACENTE ESSENTIELLE

EST UNE CONSTRUCTION SOCIALE.

Personnellement, en enseignant la philosophie des sciences et en m'affirmant moi aussi comme un partisan du constructionnisme social, j'ai été constamment confronté à des réactions similaires de la part de mes étudiants. Bien plus, un épidémiologiste spécialisé dans les morts reliées aux drogues a tenté un jour de me réduire au silence en affirmant que "un mort est un mort» et qu'il n'y a rien de socialement construit dans le fait d'être mort. Je lui ai répondu que, bien sûr, un mort était un mort, mais que nos interprétations de la mort demeuraient néanmoins une construction sociale.

Il semble bien que les partisans du constructionnisme social tentent de dire quelque chose que les autres sont incapables de comprendre sur le champ et qu'ils interprètent à tort, par conséquent, comme une négation des faits. Pour défendre le constructionnisme social, il vaut donc mieux commencer par montrer clairement que cette théorie ne nie pas les faits.

Il y a un fait que je n'entends pas nier, c'est qu'il existe des doses mortelles de substances légales et illégales. Par exemple, il existe des doses mortelles d'alcool et d'héroïne. Une dose mortelle d'alcool est aussi fatale qu'une dose mortelle d'héroïne. En ce sens, dire que les drogues tuent est un truisme. Par contre, si j'admets que les drogues tuent, pourquoi le fait de mettre une mort sur le compte de la drogue est-il une construction sociale? La réponse est la suivante: en pratique, la mort peut être attribuée à bien des causes. Réduire toutes ces causes à une seule en faisant d'une drogue illégale la cause sous-jacente essentielle est une construction sociale. Nous allons le prouver dans la section suivante avec le cas du chauffeur de taxi Taki.

\section{LE CAS}

\section{DU CHAUFFEUR DE TAXI TAKI}

Il s'agit de l'histoire d'une mort attribuée à la drogue. C'est un cas fictif, mais inspiré de la vie réelle.

Voici l'histoire. Taki naît de parents qui gagnent péniblement leur vie comme propriétaires d'un commerce de quartier. Ils sont souvent au travail et n'ont pas le temps de veiller à l'éducation de Taki. Celui-ci souffre d'un manque d'affection et acquiert peu de résistance émotionnelle et sociale. Il devient ainsi une victime toute désignée de harcèlement d'abord à l'école, puis au service militaire et finalement au travail lorsqu'il devient chauffeur de taxi. Quand l'idée du suicide l'obsède trop, Taki se libère de cette tension insoutenable au moyen de l'alcool. Cela lui permet tout juste de survivre.

Un jour, un traité supranational impose la libéralisation du marché du taxi. Pour négocier avec les clients, il faut désormais des aptitudes sociales et émotionnelles que Taki ne possède malheureusement pas. Un soir, il entend dans une conversation radiophonique un spécialiste des drogues affirmer que la cocaïne ne crée pas d'accoutumance. Il fait l'essai de cette drogue et découvre qu'elle constitue un bon moyen de combattre la migraine causée par l'alcool et de continuer à travailler. Taki se trouve donc entraîné dans le cercle vicieux de la consommation d'alcool et de cocaïne. Il boit pour trouver le sommeil la nuit et il «sniffe» de la coke pour se réveiller le matin et avoir le courage d'aller travailler.

Un jour qu'il a consommé trop de cocaïne, Taki perd toute perception juste de lui-même. Il surestime mortellement ses aptitudes en tentant de dépasser un chauffeur de taxi concurrent. Roulant trop vite dans une courbe trop serrée, il perd le contrôle de son véhicule et se tue.

\section{DE QUOI TAKI EST-IL MORT ?}

On peut prétendre que la mort de Taki n'est reliée que de loin aux drogues. Ou trouver plus percutant d'affirmer au contraire que Taki est mort d'une surdose d'alcool et de cocaïne. Quoi qu'il en soit, nous avons choisi cet exemple parce qu'il y a des pays où la mort de Taki figurera dans les statistiques officielles comme un décès dû à la drogue.

Actuellement, des dirigeants imposent une construction sociale qui fait de la cocaïne une drogue illégale. Les construc- tions sociales qui prédominent constituent la lunette à travers laquelle nous voyons le monde. Lorsque nous regardons la cocaïne à travers une lunette qui fait d'elle une drogue illégale, il ne fait aucun doute que la mort de Taki est due à la drogue. À cause de cette lunette, nous pouvons affirmer que Taki serait encore vivant s'il n'avait pas fait l'essai de la cocaïne et que, par conséquent, il est mort parce qu'il « sniffait » de la coke.

En réalité toutefois, à part d'avoir eu une mort associée à la drogue, Taki a connu mille morts. On pourrait tout aussi bien interpréter son histoire tragique comme une mort due à la négligence dans l'enfance, à de mauvais traitements, à du harcèlement à l'école, au service militaire et au travail, à la consommation d'alcool, à une émission radiophonique, à un excès de vitesse; ce pourrait être aussi un cas éloquent de mort due à une conduite à trop grande vitesse dans une courbe abrupte. Ces exemples montrent qu'une mort peut être attribuée à bien des facteurs différents.

Selon moi, Taki est en fait une victime du libre marché. C'est à cause du libre marché que ses parents devaient travailler si fort et c'est aussi à cause de la libéralisation du marché du taxi que Taki a fini par avoir un accident. Si l'on avait imposé suffisamment de restrictions sociales au libre marché dans lequel il devait vivre, Taki serait encore vivant et peut-être même heureux.

Lorsque j'essaie de présenter le concept de mort due au libre marché, on m'objecte souvent que le libre marché ne peut pas avoir causé la mort de Taki parce qu'il n'est pas reconnu comme une cause officielle de décès. On ne trouve même pas l'expression «mort due au libre marché » dans les dictionnaires. Je réponds alors que les choses qui sont reconnues officiellement et qui ont droit à une entrée dans les dictionnaires sont des constructions sociales. Mes opposants n'ont qu'à prendre mon article et qu'à l'envoyer à l'éditeur de leur dictionnaire en lui disant que son dictionnaire n'est plus du tout à la page parce qu'on n'y trouve pas encore l'expression «mort due au libre marché ».

Je sais bien que si vous ne disposez pas d'un certain mot, vous ne serez pas capable de voir ce que ce mot vous permet de voir (voir Cruts, 1991, 1994). Accepter un mot, c'est aussi accepter de regarder le monde à travers une lunette qui lui donne une certaine coloration. Par conséquent, je vous incite à ajouter dans votre dictionnaire

ACCEPTER UN MOT, C'EST AUSSI ACCEPTER DE REGARDER LE MONDE À TRAVERS UNE LUNETTE QUI LUI DONNE UNE CERTAINE COLORATION. 


\section{PARLONS UN PEU DE... CONSOMMATION DE DROGUES}

"Selon une étude effectuée auprès de la jeune génération, trois adolescents sur quatre consomment de l'alcool et six sur dix des drogues. [...] Certains consomment pour fuir leurs problèmes, combattre leur timidité ou, tout simplement, pour suivre les autres. Certains consomment uniquement pour le plaisir de la chose. [...] Pour certaines personnes, la timidité est pratiquement un problème chronique. Alors, si l'on ajoute à ce problème un entourage qui consomme (et donc de la marchandise facile d'accès), qu'est-ce qui en résulte? Une solution qui paraîtra des plus simples à toute personne désespérée: la drogue. Après tout, les drogues (du moins les douces) ne sont-elles pas reconnues pour leurs facultés socialisantes? [...] Un adolescent qui fréquente de jeunes toxicomanes finit toujours par se retrouver devant un choix: changer de bande ou s'y mettre lui aussi."

Benoît Trépanier, Amélie Choquette, Jonathan Pineault et Sébastien Roy

personnel l'expression «mort due au libre marché ». En étant bien au courant de ce type de mort, vous éviterez peut-être d'en être vous-même victime.

\section{UN RAISONNEMENT INVÉRIFIABLE DANS LES FAITS}

Pour essayer de prévenir les morts dues au libre marché, j'affirme que si l'on imposait des restrictions sociales au libre marché, moins de personnes en mourraient. Mon raisonnement est souvent contré par l'objection suivante: «Vous raisonnez ainsi parce que vous êtes un socialiste. » Je ne peux que répondre que je suis en effet un socialiste. C'est en tant que socialiste que je blâme le libre marché pour la mort tragique de Taki. Un libéral, par contre, mettrait cette mort sur le compte de la cocaïne en disant que Taki ne serait pas mort si la cocaïne n'avait pas existé.

Un raisonnement qui se construit de la façon suivante: "s'il n'y avait pas eu A, il n'y aurait pas eu B» est la contreproposition qui permet de valider un raisonnement en montrant que, sans les faits de départ, la conclusion aurait été différente. La discussion sur la cause de la mort de Taki montre que le fait d'attribuer cette mort à une certaine cause doit, pour être valide, s'appuyer sur une proposition contraire. Quelque chose est considéré comme la cause de la mort de Taki parce que sans ce «quelque chose» Taki ne serait pas mort.

Le problème philosophique que pose cette contre-proposition est que sa véracité ne peut jamais être prouvée empiriquement (Cruts, 1993). En d'autres mots, les raisonnements sur ces contre-propositions sont invérifiables. Rien ne peut les confirmer ou les infirmer. Il va de soi qu'une personne qui est encore vivante n'a pas pris une dose mortelle de drogue. C'est une vérité logique, mais son contraire n'est pas nécessairement vrai. Une personne qui n'a pas pris une dose mortelle de drogue n'est pas nécessairement encore en vie, parce qu'il y a bien des façons de mourir.
S'il n'y avait pas de doses mortelles de cocaïne, que serait devenu Taki? Il serait peut-être encore vivant, mais il aurait pu également se suicider parce qu'il ne réussissait pas dans son travail. La cocaïne a peut-être retranché bien des années de sa vie, mais elle a pu également lui en ajouter quelques-unes. Ce qui est absurde, c'est que la chose qui nous garde en vie peut aussi nous tuer (voir Cruts, 1995). Pour qu'il puisse demeurer en vie sans consommer de cocaïne, il aurait fallu que Taki trouve d'autres moyens de surmonter le stress relié à son travail. La seule solution aurait peut-être été de changer d'emploi. Il aurait peut-être fait un bon chauffeur d'autobus. Les faits ne nous diront jamais si cela aurait pu survenir dans la vie d'un Taki ne consommant pas de cocaïne.

À l'école primaire, on m'a montré des peintures de Rembrandt et de Van Gogh et, tout jeune, je me suis demandé lequel des deux peignait correctement. Je ne comprenais pas pourquoi l'institutrice ne nous disait pas quel peintre donnait une représentation juste des faits. Devenu adulte, je comprends maintenant que les faits ne peuvent pas nous dire qui de Rembrandt ou de Van Gogh est le meilleur peintre. Le but d'un peintre n'est pas de donner une représentation juste des faits, mais de jeter une lumière nouvelle sur eux.

De même que les faits ne permettent pas de trancher empiriquement entre Rembrandt et Van Gogh, ils ne permettent pas non plus de trancher au sujet de la question de la mort attribuée à la drogue. Attribuer une mort à une drogue ou à une autre cause comme le libre marché, c'est s'appuyer sur un raisonnement qu'on ne peut valider. Ne pouvant jamais être prouvé empiriquement, ce genre de raisonnement exprime notre philosophie de la vie.

Différentes opinions au sujet des drogues expriment souvent différentes philosophies de la vie. Tenter de convaincre les autres du bien-fondé de sa propre philosophie à l'aide de faits est frustrant pour les deux parties. Si ces faits ne peuvent mettre fin à la controverse, il est inutile de s'évertuer à les rechercher.

$\mathrm{Au}$ lieu de réunir de plus en plus de faits au sujet des morts attribuées à la drogue, il vaut mieux nous dire mutuellement sous quel éclairage nous regardons les faits. Nous ne pouvons que nous inviter les uns les autres à regarder le monde à travers une certaine lunette. Je n'ai fait que vous suggérer d'adopter le point de vue du constructionnisme social pour regarder un sujet aussi grave que la mort attribuée à la drogue.

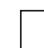 \\ PARLONS UN PEU... DES DROGUES \\ «Plusieurs jeunes désirent découvrir leur identité dans l'interdit. La drogue, étant prohibée, les séduit par son côté défendu. [...] La "seule façon" pour ces gens en détresse de s'évader, est d'avoir recours à la drogue vendue par des trafiquants, souvent dangereux et pas très recommandables. La légalisation des matières illé- gales permettrait aux toxicomanes de vivre avec des gens honnêtes qui souhaitent les voir guérir et revenir sur le droit chemin. [...] Le désir d'obtenir le monopole de la vente des drogues pousse les bandes criminalisées rivales à se faire une guerre de rue sans pitié. Dans les grandes villes du Québec, on assiste à des fusillades, des attentats, des meurtres... Des innocents périssent dans cette guerre de pouvoirs. » \\ «Mais comment pouvez-vous même y songer? La consommation après la dérégle- mentation du commerce de la drogue ne pourra que s'accroître! À chaque coin de rue, les drogués pourront se procurer des produits illicites, à de meilleurs prix et de meilleure qualité. De plus, que faites-vous des mortalités causées par les buveurs excessifs? Les mauvais traitements infligés par ceux-ci? N'est-il pas logique de penser qu'à la légalisation des drogues, les dégâts ne pourront que doubler? [...] Le problème n'est pas la diminution ou l'augmentation de la consommation, mais le fait que les gens sous l'effet des drogues ou de l'alcool fassent porter des coûts à d'autres individus sans que ceux-ci y aient consenti. » \\ Myriam Cyr, François Lachance, Éric De Lafontaine et Marc-André Dufrene}




\section{Bibliographie}

COHEN, D.A.P. (1990). Drugs as a Social Construct, Amsterdam, Elsevier.

CRUTS, A.A.N. (1995). «The absurd in psychology ", dans I. LUBEK, R. VAN HEZEWIJK, G. PHETERSON et C. TOJMAN (dir.), Trends and Issues in Theoretical Psychology, New York, Springer, p. 373-375.

CRUTS, A.A.N. (1993). «Possible lives: the case of unfalsifiable modal cognitions", Cognitive Systems, no 3, p. 68-80.

CRUTS, A.A.N. (s.d.). "The influence of folk psychology on a baby's personality " dans J.J.F. TER LAAK, P.G. HEYMANS et A.J. PODOLSKIJ (dir.), Developmental Tasks: Towards a Cultural Analysis of Human Development, Dordrecht, Kluwer, p. 107-124.

CRUTS, G. (1991). "The language games of child development: a search for tools to represent parental cognitions", Cognitive Systems, n 3, p. 21-42.
DAVIES, J.B. (1997). Drugspeak: The Analysis of Drug Discourse, Amsterdam, Harwook Academic Publishers.

DeGRANDPRÉ, R.J. (1996). "The impact of socially constructed knowledge on drug policy », dans W.K. BICKEL. et R.J. DeGRANDPRÉ (dir.), Drug Policy and Human Nature Psychological Perspectives on the Prevention, Management and Treatment of Illicit Drug Abuse, New York, Plenum, p. 301-322.

GERGEN, K.J. (1999). An Invitation to Social Construction, Londres, Sage Publications.

LEGNARO, A. (1997). «Drogen-Tod: Die empirische Realität eines sozialen Konstrukts », BINAD-Info, no 8, p. 9-11.

LYONS, P. et RITTNER, B. (1998). "The construction of the crack babies phenomenon as a social problem », American Journal of Orthopsychiatry, $\mathrm{n}^{\circ} 68$, p. 313-320.
SCHNEIDER, W. (1996). "Der gesellschaftliche Drogenkult: Essays zur Entzauberung von Drogenmythen», dans Drogenhilfe, Drogenforscchung und Drogenpolitik, VWB für Wissenschaft und Bildung, Berlin.

Van de WIJNGAART, G.F. (1991). Competing Perspectives of Drug Use (The Dutch Experience), Amsterdam, Lisse, Swets et Zeitlinger.

\section{Notes}

1. Ce texte est paru en anglais sous le titre "The social construction of drug-related death », dans International Journal of Drug Policy, vol. 11, n 6, 2000, p. 381-385.

2. Suzanne Mineau est une traductrice professionnelle qui se spécialise en sciences humaines. Elle est membre de l'Association des traducteurs et traductrices du Canada ainsi que de l'Ordre des traducteurs, terminologues et interprètes agréés du Québec.

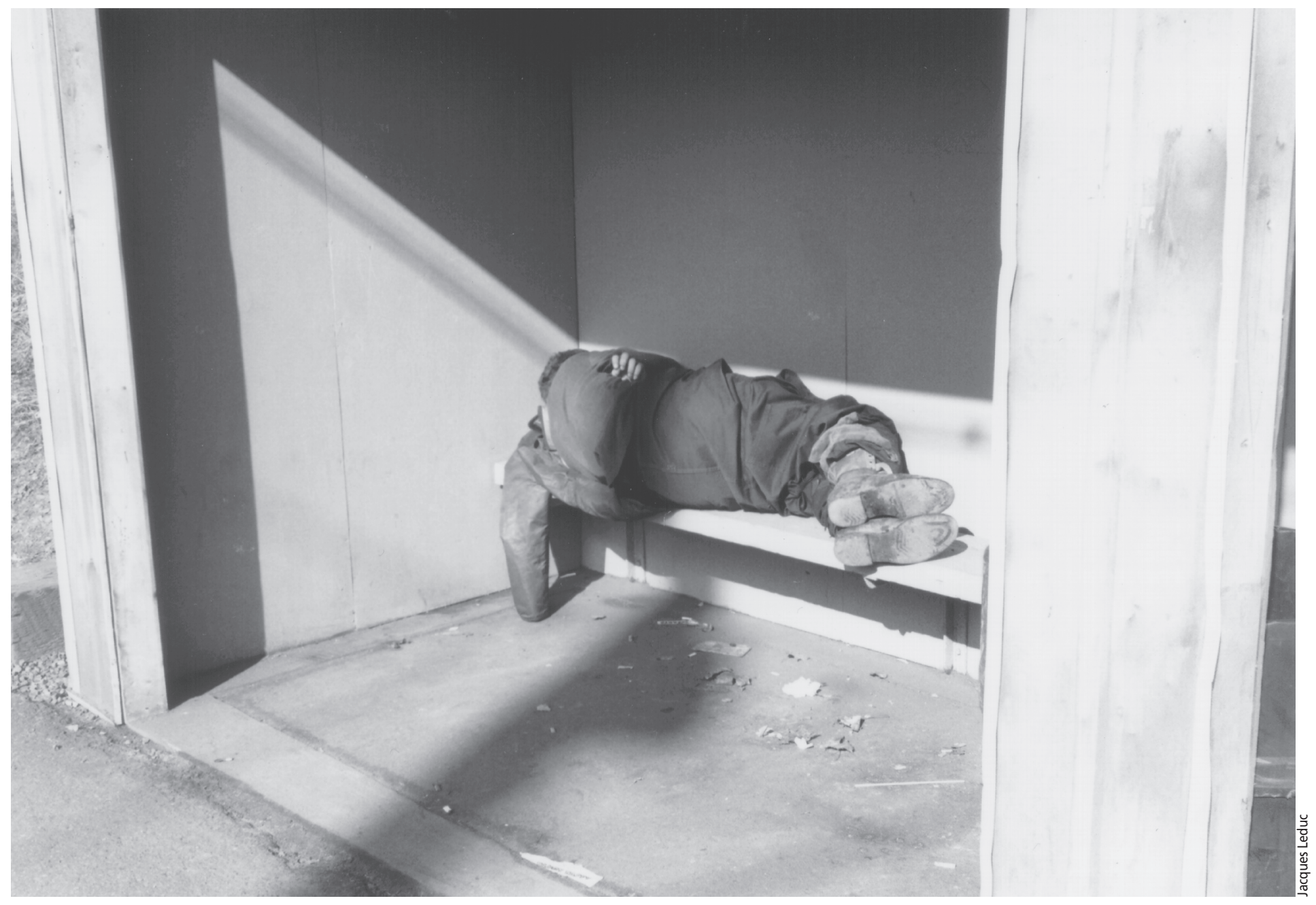

